\title{
Research on the Wisdom Symbiosis of Learners Based on Cloud Course Platform
}

\author{
Jinzhong Ma \\ Teachers Education College \\ YanBian University \\ Yanji, China 133002
}

\author{
Yuhua Jiang* \\ Baicheng Normal College \\ Baicheng, China 137000 \\ *Corresponding Author
}

\begin{abstract}
With the development of Internet plus education, a variety of learning methods and learning resources platform have been produced. Among them, the Cloud course platform is a typical example. The cloud curriculum platform is a kind of learning carrier formed by the combination of Internet technology and mobile terminal. It has the characteristics of openness and interactivity, which meets the needs of individualized learning and cooperative learning, and has a significant impact on learners' learning. This paper studies the research of the cloud curriculum platform in recent years through the literature method, and the researchers think that the Could course platform has a great effect on the learners' learning habits, learning styles and learning interests, but quite a few students Self-learning awareness is not strong, lack of learning ability, which requires teachers to be facilitators of a new concept of learning, learners to form an effective learning community, establish active learning awareness so as to effectively improve the application of Cloud course platform, to improve the students' informationalized ability, to achieve the depth development of learners wisdom.
\end{abstract}

Keywords—cloud course platform; individualized learning; cooperative learning; wisdom symbiosis

\section{INTRODUCTION}

In recent years, the development of cloud environment has brought new ways of education and learning, internet courses, MOOC, cloud course platform and so on, they provide learners with more convenient ways to learn. In 2012, the Ministry of National Education put forward "to establish the national education cloud service model, fully integrate existing resources, use cloud computing technology to form the intensive development path of resource allocation and service, and build a stable, reliable, low-cost national education cloud service model" in the Ten Year Development Plan of Educational Informatization (2011-2020). The learning of the cloud course platform support has become the trend of development of the time. Under the impact of new knowledge concept, leaning concept and talent concept, the static accepted-style learning with simple knowledge transfer has been shaken, more and more attention has been attracted to the personalized leaning based on intelligence generation. The learning environment built by the cloud course platform is one of the important ways to realize the wisdom generation.

In the research on the cloud course platform from home and abroad, most of them are the development and design of the cloud course platform, however, there are few researches on effective utilization of cloud course platform. This study helps learners to realize the resource sharing and wisdom symbiosis by exploring ways to rationally and effectively apply the cloud course platform, and helps learners to establish learning awareness of using the cloud course platform, so as to make learners become masters of digital learning era.

\section{CURRENT Research OF THE Cloud COURSE PLATFORM AND WISDOM GENERATION}

In May 2006, "cloud computing" was first proposed by Google, cloud computing took the lead in the rapid rise of business; subsequently, cloud computing went into the education domain, and there was a cloud course platform, of which the Google and Microsoft cloud platforms provided teachers with a large number of teaching resources and software applications to help teachers carry out various teaching activities. Cloud course platform has been a trend of education development. It is a kind of learning carrier which combines Internet technology with mobile terminal. Pan Xinmin mentioned that "The cloud curriculum is a stable and sustainable sharing platform that can vigorously promote education informationization and implement education resource sharing", and he said "the Cloud curriculum is a new curriculum form based on new technologies such as cloud technology(cloud computing)and wisdom mobile. Specifically, it's a new curriculum form which takes the cloud platform as the curriculum carrier, takes the educational target, subject knowledge, relevant curriculum resources and the expected teaching activity way as the course content by an organic coordination and integration of them, takes the combination of formal learning with informal learning and of massed learning with personalized learning as the main way of curriculum teaching"[2], and as the carrier of new curriculum form, the cloud course platform has created a diversified and open information exchange platform for learners. Taking NetEase cloud platform as an example[3]; Luo Xianghui and Liu Yun introduced the advantages of cloud course platform and the way to open online courses[4]; Wang Jing and Yang Zhuo, taking the cloud class of Central China Normal University as an example, discussed the blended instructional design model in the cloud class.

In generative learning, there are more researches on generative learning resources in recent years. Wan Liyong, et 
al expounded the origin and characteristics of generative learning resources from the perspective of generative resources; Yang Xianmin and Yu Shengquan studied the evaluation index design of generative learning resources; He Xiangyang and Xiong Caiping discussed the relevant attributes and models of generative learning resources. On the whole, the study of generative learning focuses on the related principles of generative learning.

The generative learning is based on the constructivist learning theory, emphasizing the collaborative construction and dynamic generation of knowledge, emphasizing the deep thinking, communication and interaction of students. American educator John Dewey believes that wisdom is the various capabilities to use knowledge to guide life and improve life, so wisdom generation has gone beyond the independent construction of knowledge, but also the dynamic generation of various capabilities. In the information age, wisdom generation is the "symbiosis transition" that enables learners to achieve burst wisdom from the sharing learning" based on data, and the wisdom symbiosis is the wisdom generation of the group.

\section{RESOURCE SHARING AND WISDOM SYMBIOSIS BASED ON THE CLOUD COURSE PLATFORM}

Interactivity is a typical feature of learning in the cloud environment, resource sharing is the core of cloud platform learning, the open resources in the cloud course platform create the conditions for the wisdom symbiosis. Through the integration and reorganization of various resources, learners can inspire and arouse the resonance of thinking under the interaction of multiple levels. Among them, there are three different levels of interaction in the cloud course platform, and the most basic is the interaction between the learner and the cloud course learning platform and the media, namely humancomputer interaction. The interaction of this level depends more on the development and construction effect of the cloud course platform. This interaction is mainly the query and acquisition of resources by learners using the navigation design of the platform. The second level of interaction is the interaction between the realization and learning resources, companions, teachers, and so on, and the interaction of this level is the process of helping learners to integrate into the learning community, namely human-human interaction. This process is the active learning of learners and resource sharing between learning community; the third interaction is the requirement of the highest level for learners, the learner's individual wisdom, knowledge and constantly updated contact interaction, namely the self-sublimation interaction.

First course platform can provide students with vast amounts of resources, so that the students in the network environment to realize anyone at any time, place to get resources, so that resource sharing to a certain extent, but it ignores the connection between individual knowledge of learner and public knowledge, and does not realize the true meaning of wisdom generation. According to the hierarchical structure model of DIKW (Data, Information, Knowledge, Wisdom)proposed by Thomas Stern Eliot, from resource sharing to symbiotic wisdom generation system, it focuses on the evolution of "data - information-knowledge-wisdom" of individuals and groups. Cloud curriculum platform pays attention to the multiple interactions that highlight the learner's dominant position, which is conducive to the realization of wisdom symbiosis. Learners get resources from the platform according to their own needs. According to Piaget's cognitive development theory, learners on the basis of the original knowledge and ability, namely on the existing cognitive "schemata", after "adaptation" and" assimilation", produce the connection between the old and new knowledge, to achieve a "balanced" state, completed the process of learner's personal knowledge building; generative learning theory proposes that the formation process of personal knowledge is not only the link between personal knowledge and personal knowledge, but also the link between personal knowledge and public knowledge. The link of personal knowledge should be externalized expression, communicated, discussion, activity experience and reflection internalization so as to realize the renewal and generation of personal knowledge in the true sense. Learners use self-constructed new knowledge to create and express themselves in the learning community, through sharing, feeling, experience and reflection, find new problems, trigger a deeper thinking, at this time, the knowledge of personal construction has been sublimated into wisdom, the wisdom as renewable resources continue to share in the learning community, realize the generation of swarm wisdom. The learner's wisdom symbiosis process diagram is based on the cloud course platform.

\section{EFFICIENT WAYS OF REASONABLE APPLICATION OF THE CLOUD COURSE PLATFORM}

According to the results of the survey, the learning based on the cloud course platform is of great positive significance to the learner's wisdom generation, but the overall effect needs to be improved. The effective way of reasonable application of cloud course platform is put forward from the point of teachers and students respectively.

\section{A. Teachers Should Be the Facilitators and Guides of New Learning Concepts and Learning Styles}

From the results of the study, it is not difficult to find, for the new learning tools and learning methods, students need the guidance of teachers, teachers in the classroom active use will greatly help students improve learning effect, enhance the ability, and realize the wisdom generation. Each teacher has his own unique teaching style. The uniqueness of this teaching is the teachers' intrinsic Individual Property, which is called Teaching IP. However, the difference of Teaching IP does not affect teachers' improvement of students' learning style. In a cloud environment, the Teaching IP need to constantly inject fresh blood, the student to the new things understanding and application needs a process of accept, in the primary stage to accept new things, inevitably encounter all sorts of problems, at this point, the teachers' guide and promote is particularly important.

\section{B. Teachers Should Be Effective Organizers of Learning Activities and Carry out Accurate Teaching for Different Grade Students}

The results show that there is no significant gender difference in the learning of the cloud course platform, but 
there are obvious differences in different grades. In different grades, the organization of learning activities should be targeted, and students in lower grades need teachers to supervise and guide them in a timely manner due to the lack of knowledge and learning ability. The study of the super nebula curriculum platform has realized the multiple interactions of learners, so that everyone can participate in the study and complete the study in the communication and collaboration. It takes more account for the stimulation of students' learning motivation, the diversity of student' evaluation, the students' emotional experience and the generation of students' ability and wisdom.

\section{Learners should Build an Effective Learning Community}

The research shows that most of the students use the course learning platform because of teacher's recommendation, the use of teachers' classroom prompts their application of cloud course learning platform, and only few students apply it in order to meet the requirements of their own learning. It can be seen that some students are not aware of active learning and have not formed a good sense of autonomous learning. It can be seen that some students have a weak awareness of active learning and have not formed a good sense of autonomous learning, and their thinking and habits remain in the traditional teaching of teachers. In the digital age, a wealth of learning resources is to meet the needs of personalized learning. We have reached a high consensus that innovation is indispensable for education in the future. In the concept of generative learning, and under the concept of generative learning, classroom learning is only part of our learning, and the combination of traditional learning and online autonomous learning can better promote the dynamic generation of knowledge. At present, all kinds of MOOC learning platform, a variety of the cloud courses learning platform, mobile learning APP and WeChat public number and other learning carriers provide more possibilities and opportunities for our study. We should have the ability not only to choose the learning carrier, but also to collect and identify useful information from the mass of learning resources, which is also a kind of literacy for learners in the digital information age. We should make students develop good learning habits and become the master of the digital age.

In the learning based on the cloud course platform, we can readily find that it achieves multiple interactions; by online, it includes the navigation interaction between learners and the cloud courses learning platform, the interaction among learners and peers and teachers; and the process of acquiring resources is the process of human-computer interaction; by offline, it including in the classroom, it can achieve face-to-face interaction among students and among students and teachers; In the process of interaction, the effective allocation of resources is realized, and the resource is regenerated in the process of utilization and sharing so as to realize the wisdom symbiosis.

\section{CONCLUSION}

The cloud course platform provides a platform for learners to gather curriculum resources, learners resources and learners space. Each learner and instructor can communicate with each other through their own mobile terminals, share experience and answer questions. The learning based on the cloud course platform fully exerts everyone's participation in learning, and everyone is the master of learning. The teachers, students and peers are skillfully interconnected through various interactions to form a virtual learning network. In this network, each node is a learner, each line is the communication between learners; each space formed by points and lines is the wisdom resources generated. Therefore, only by constantly optimizing their learning methods, learners can continue with the learning system network, so as to realize the interaction of learners with peers and teachers, and to get the wisdom symbiosis in the process of sharing.

\section{REFERENCES}

[1] The Ministry of National Education. Ten Year Development Plan of Educational Informatization(2011-2020) [R]. 2012, 3

[2] Pan Xinmin. “Cloud Courses” :Characteristics, Significance and Problems [J]. Curriculum Textbook Pedagogics, 2013,(12):8-12

[3] Liu Shaojuan. Cloud Courses: the Pursuit of Personalized Education in the Era of Big Data [J]. Modern Education Science, 2015, (06):135-137

[4] Luo Xianhui, Liu Yun, Kang Xiao Li. Online Open Courses Based on the Free Cloud Courses Platform: ACase Study of "NetEase Cloud Courses", [J] Information Construction, 2015, (11): 160-161

[5] E.H.Chi. A Taxonomy of Visualization Techniques Using the Data State Reference Model, Proc. IEEE Symposium on Information Visualization[C]. Salt Lake City, Utah, 2000: 69-75. 